6. K. Iwasawa, Über die Einfachheit der speziellen projectiven Gruppen, Proc. Imp. Acad. Tokyo vol. 17 (1941) pp. 57-59.

7. F. A. Lewis, The linear congruence group modulo n, Proc. Amer. Math. Soc. vol. 3 (1952) pp. 367-368.

8. B. L. van der Waerden, Gruppen von linearen Transformationen, Berlin, Springer, 1935.

9. S. Wang, On the commutator group of a simple algebra, Amer. J. Math. vol. 72 (1950) pp. 323-334.

UNIVERSITY OF CONNECTICUT

\title{
A NOTE ON INVARIANT SUBRINGS
}

\section{GERTRUDE EHRLICH}

The problem of invariant subrings has been studied in detail for certain types of rings satisfying the descending chain condition on one-sided ideals.

We prove here two theorems concerning invariant subrings of a ring $R$ without assuming the descending chain condition.

THEOREM 1. Let $R$ be a ring with identity 1 . If $S$ is a subring of $R$ with identity 1 , and $S$ has a representation as the complete matrix ring of order $n \geqq 2$ over a ring with identity, then $S$ cannot be a proper invariant subring of $R$.

Proof. Let $E=\left\{e_{i j}\right\}$ be a set of $n^{2}$ matrix units contained in $S$, and let $B$ be the centralizer of $E$ relative to $S$. Then $S=B_{n}$. Let $A$ be the centralizer of $E$ relative to $R$. Then $R=A_{n}$, and $B \leqq A$. If $t$ is an arbitrary element of $A$, we obtain (following Hattori [1]) that $e_{i i}\left(1+e_{i j} t\right) e_{j i}\left(1-e_{i j} t\right) e_{i i}=t e_{i i}$ belongs to $S$ for arbitrary $i \neq j$. Hence $t=\sum_{i=1}^{n} t e_{i i}$ belongs to $S$, and $A \leqq S$. But then $A=B$, and $S=R$.

THEOREM 2. Let $R$ be a ring with identity 1 , and not of characteristic 2. Assume that $R$ has a representation as the complete matrix ring of order $n \geqq 2$ over a ring with identity. Let $S$ be an invariant sub-sfield of $R$, with identity 1. Then $S$ is a subfield of the center of $R$.

Proof. Let $E=\left\{e_{i j}\right\}$ be a set of $n^{2}$ matrix units contained in $R$, and let $A$ be the centralizer of $E$ relative to $R$, so that $R=A_{n}$. We note first that for every noncentral element $x$ contained in $R$, there exists a square-nilpotent element $p \neq 0\left(p^{2}=0\right)$ such that $x p \neq p x$. (For, if $x$ commutes with every square-nilpotent element, then $x$ commutes in

Received by the editors, August 9, 1954. 
particular with the elements $e_{i j}$ and $e_{i i} y e_{j j}$ for $i \neq j$, and $y$ arbitrary in $R$. This implies that $x$ belongs to the center of $R$.) Now, suppose $S$ is an invariant sub-sfield of $R$, and $1 \in S$. If $S$ contains a noncentral element $x$, then $R$ contains a square-nilpotent element $p \neq 0$ such that $x p \neq p x$. Let $t=2+p$. Then $t^{-1}$ and $(t-1)^{-1}$ belong to $R$. Using Hua's identity [2]

$$
t=\left[x^{-1}-(t-1)^{-1} x^{-1}(t-1)\right]\left[t^{-1} x^{-1} t-(t-1)^{-1} x^{-1}(t-1)\right]^{-1}
$$

and the hypotheses on $S$, we conclude that $t$ belongs to $S$. But then $p$ belongs to $S$. This is impossible since $p$ can have no inverse relative to the identity 1 .

\section{REFERENCES}

1. A. Hattori, On invariant subrings, Jap. J. Math. vol. 21 (1951-52).

2. L. K. Hua, Some properties of a sfield, Proc. Nat. Acad. Sci. U.S.A. vol. 60 (1946).

UNIVERSITY OF MARYLAND 